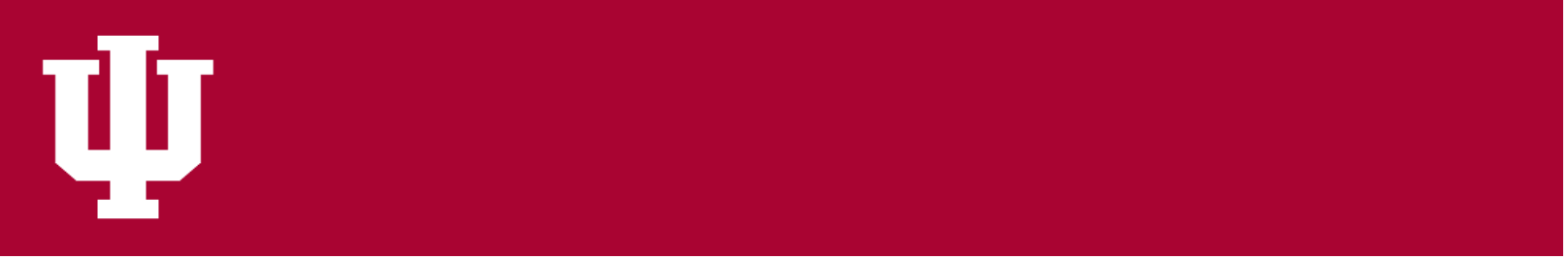

IUScholarWorks at Indiana University South Bend

\title{
Modulation of Appetite and Feeding Behavior of the Larval Mosquito Aedes Aegypti by the Serotonin-Selective Reuptake Inhibitor Paroxetine: Shifts between Distinct Feeding Modes and the Influence of Feeding Status
}

Kinney, M. P., Panting, N. D., \& Clark, T. M.

To cite this article: Kinney, Michael P., et al. "Modulation of Appetite and Feeding Behavior of the Larval Mosquito Aedes Aegypti by the Serotonin-Selective Reuptake Inhibitor Paroxetine: Shifts between Distinct Feeding Modes and the Influence of Feeding Status." Journal of Experimental Biology, vol. 217, no. 6, Mar. 2014, pp. 935-43. doi:10.1242/jeb.094904.

This document has been made available through IUScholarWorks repository, a service of the Indiana University Libraries. Copyrights on documents in IUScholarWorks are held by their respective rights holder(s). Contact iusw@indiana.edu for more information. 


\title{
Modulation of appetite and feeding behavior of the larval mosquito Aedes aegypti by the serotonin-selective reuptake inhibitor paroxetine: shifts between distinct feeding modes and the influence of feeding status
}

\author{
Michael P. Kinney, Nicholas D. Panting and Thomas M. Clark*
}

\begin{abstract}
The effects of the serotonin-selective reuptake inhibitor paroxetine $\left(2 \times 10^{-5} \mathrm{moll}^{-1}\right)$ on behavior of the larval mosquito Aedes aegypti are described. Four discrete behavioral states dominate larval behavior: wriggling, two distinct types of feeding, and quiescence. Feeding behaviors consist of foraging along the bottom of the container (substrate browsing), and stationary filter feeding while suspended from the surface film. Fed larvae respond to paroxetine with increased wriggling, and reductions in both feeding behaviors. In contrast, food-deprived larvae treated with paroxetine show no change in the proportion of time spent wriggling or feeding, but shift from stationary filter feeding to substrate browsing. Thus, actions of paroxetine in fed larvae are consistent with suppression of appetite and stimulation of wriggling, whereas paroxetine causes fooddeprived larvae to switch from one feeding behavior to another. Further analysis of unfed larvae revealed that paroxetine decreased the power stroke frequency during wriggling locomotion, but had no effect on the swimming velocity during either wriggling or substrate browsing. These data suggest that: (1) serotonergic pathways may trigger shifts between distinct behaviors by actions on higher level (brain) integrating centers where behaviors such as feeding and locomotion are coordinated; (2) these centers in fed and fooddeprived larvae respond differently to serotonergic stimulation suggesting sensory feedback from feeding status; and (3) serotonergic pathways also modulate central pattern generators of the nerve cord where the bursts of action potentials originate that drive the rhythmic muscle contractions of wriggling.
\end{abstract}

KEY WORDS: Aedes aegypti, Mosquito, Behavior, Paroxetine, Serotonin, Feeding, SSRI, Appetite, Wriggling, Substrate browsing, Stationary filter feeding

\section{INTRODUCTION}

Animals with relatively simple behaviors switch between discrete behaviors in response to internal and external sensory cues. For example, the transition from locomotion to feeding is modulated by external stimuli associated with the presence or absence of food, and internal stimuli associated with feeding or nutritional status. Serotonergic pathways are particularly important in regulation of appetite and behavior in diverse animals (Gruninger et al., 2007; Waggoner et al., 1998; Tecott, 2007; Nässel and Wegener, 2011;

Department of Biology, Indiana University South Bend, 1700 Mishawaka Avenue, South Bend, IN 46634-7111, USA.

*Author for correspondence (tclark2@iusb.edu)

Received 31 July 2013; Accepted 5 November 2013
Luedtke et al., 2010; Gaudry and Kristan, 2012). We investigated these pathways further using the serotonin-selective reuptake inhibitor (SSRI) paroxetine $\left(\right.$ Paxil $\left.^{\mathrm{TM}}\right)$.

\section{Behavior of larval mosquitoes}

Mosquito larvae have relatively simple, species-specific behavior that includes wriggling and distinct modes of feeding (Merritt and Cummins, 1984; Hocking, 1953; Jackson, 1953; Yee et al., 2004). Wriggling is characterized by rhythmic lateral flexion of the body to alternate sides, and has been described elsewhere (Strickman, 1989; Brackenbury, 2001). Movement through the environment also occurs during feeding. Brushes of labial setae act as paddles, creating currents that draw the larva through the water (Brackenbury, 2001; Christophers, 1960; Hocking, 1953; Merritt and Cummins, 1984). Christophers termed all such mouthpart-driven movements 'browsing' (Christophers, 1960). However, two apparently distinct types of mouthpart-driven movements are described. Brackenbury described 'gliding' by Culex pipiens larvae, which occurred during filter feeding at various levels in the water column and almost always results in a straight line of travel (Brackenbury, 2001). Hocking described 'browsing' as a specific type of mouthpart-driven movement in which larvae, filter feeding while suspended from the surface by their respiratory siphons, move gradually through the habitat (Hocking, 1953). Both gliding and browsing, as described by Brackenbury and Hocking, appear to be incidental consequences of feeding rather than directed movement through the environment (i.e. locomotion) (Brackenbury, 2001; Hocking, 1953). In addition to filter feeding, larval mosquitoes also feed by scraping the substrate, and can feed on larger food sources, including dead mosquito larvae, within their habitat (Bara et al., 2014).

\section{Serotonin and behavior}

Serotonin acts both centrally and peripherally in diverse aspects of physiological homeostasis, and in modulation of diverse behavioral states, in a wide variety of animals. Central serotonergic pathways are strongly associated with appetite and satiation, and play an important role in the integration of feeding with other behavioral states including locomotion, vigilance, aggression and reproduction (Gruninger et al., 2007; Il-Han et al., 2010; Huber et al., 1997; Anstey et al., 2009; Thamm et al., 2010; Guler and Ford, 2010; Gaudry and Kristan, 2012). Regulation of appetite and feeding by central serotonergic pathways appears to be an ancestral condition among bilateral animals. In leeches and gastropods (Lophotrochozoa), serotonergic pathways in the CNS stimulate appetite, triggering shifts from other behavioral states to feeding (Gillette, 2006; Gaudry and Kristan, 2012). In these animals, satiation primarily occurs in response to sensory input from gut 


\begin{tabular}{|ll|}
\hline List of $\mathbf{a b b r e v i a t i o n s}$ \\
CNS & central nervous system \\
LED & laser emitting diode \\
SFF & stationary filter feeding \\
SSRI & serotonin-selective reuptake inhibitor. \\
\hline
\end{tabular}

stretch receptors, rather than signals reflecting the status of nutrient stores (Gillette, 2006). In the nematode Caenorhabditis elegans (Ecdysozoa), serotonin triggers a shift from roaming behavior, characterized by relatively rapid and linear movement through the environment, to dwelling behavior, characterized by frequent stops and reversals of direction resulting in the animal remaining in the same general area. At the same time, serotonin also stimulates pharyngeal pumping. These actions are consistent with stimulation of appetite and feeding behavior (Shtonda and Avery, 2006; Tecott, 2007). Serotonin also modulates insect feeding behavior. Serotonergic systems inhibit feeding in the locust, the ant, adults of two species of fly, an aphid, a cockroach and larval Drosophila (Dacks et al., 2003; Kaufmann et al., 2004; Falibene et al., 2012; Neckameyer, 2010), although serotonin appears to stimulate feeding by the adult mosquito Aedes triseriatus (Novak and Rowley, 1994). As in vertebrates, serotonergic pathways inhibit appetite and feeding behavior in insects (Gillette, 2006; Halford et al., 2007). In both insects and vertebrates, feedback to feeding centers reflecting overall energy and nutrient balance is provided by peptide hormones and neurotransmitters including neuropeptide $\mathrm{Y}$ and insulin-like peptides (Gruninger et al., 2007; Nässel and Wegener, 2011; Tecott, 2007; Luedtke et al., 2010).

The present study utilized video analysis and position tracking software to describe the behavior of the larval mosquito Aedes aegypti (L.). The serotonin-selective reuptake inhibitor paroxetine triggers shifts among several discrete behavioral states suggesting that serotonergic pathways act centrally to modulate appetite, feeding behavior and locomotion, and the actions of paroxetine are strongly influenced by physiological and sensory cues associated with feeding status. In addition, evidence is presented for serotonergic modulation of central pattern generators during episodes of wriggling locomotion.

\section{RESULTS}

\section{Behavior of larval mosquitoes}

Larvae showed a very simple behavioral repertoire during the assays, consisting of episodes of locomotion including bouts of wriggling ranging from a few to many power strokes, interspersed with episodes of mouthpart-driven locomotion, episodes without locomotion, and brief episodes of grooming of the caudal region using the mouthparts. Episodes without locomotion can be differentiated into two distinct behavioral states. During some episodes, larvae suspended from the surface film were clearly filter feeding as they flexed their bodies, moving their heads slowly from side to side, with distinct and characteristic movements of the head and mouthparts. This behavior is subsequently termed 'stationary filter feeding' (SFF). Larvae were also observed performing neither feeding nor powered movement, either at the surface or for brief periods while submerged, immediately following a bout of wriggling. Larvae not engaged in feeding, grooming, or powered movement are termed quiescent.

\section{Locomotion}

Movement around the arena occurred in two distinct behavioral states, wriggling and mouthpart-driven locomotion during foraging.

\section{Wriggling}

During wriggling, rhythmic lateral flexion of the body alternating between the left and right sides propel the larva through the water by a series of power strokes. Velocity alternated between high and low values, coinciding with the power stroke and inter-stroke recovery period. Episodes of wriggling were readily discernible in plots of the $x-y$ coordinates during the trial (Fig. 1A), as well as in plots of velocity versus time (Fig. 1B). The Swistrack tracks during episodes of wriggling across the center of the arena consisted of a series of relatively straight, high velocity segments during power strokes, separated by low velocity shifts in the direction of travel that occur between power strokes (Fig. 1C). Velocity during wriggling averaged $11.2 \pm 1.48 \mathrm{~mm} \mathrm{~s}^{-1}$, but ranged from 1 to $4 \mathrm{~mm} \mathrm{~s}^{-1}$ between power strokes to an apparent maximum velocity of $20-35 \mathrm{~mm} \mathrm{~s}^{-1}$ during the power strokes (Fig. 1B,D, Table 1). Frame rate and tracking limitations precluded more accurate estimates of maximum velocity during wriggling power strokes.

\section{Mouthpart-driven locomotion during foraging}

Larvae in these experiments spent significant time engaged in foraging along the bottom of the arena, with their movements powered by rhythmic actions of the labial brushes. This distinct behavior was clearly different from the mouthpart-driven movements described previously. The behavior described as gliding (Brackenbury, 2001) consists of mouthpart-driven movements associated with filter feeding, during which the larvae always travel in a straight line, suggesting that the movement is not directed but is instead a passive consequence of filter feeding. Gliding occurs at any level of the water column and is not limited to the bottom (Brackenbury, 2001). It also differs from the behaviors termed browsing (Christophers, 1960; Hocking, 1953). Browsing as defined by Christophers consists of all mouthpartdriven movements, and would include gliding (Christophers, 1960). Browsing as described by Hocking (Hocking, 1953), is the gradual movement across the habitat of larvae that are filter feeding while suspended from the surface film, and like gliding does not appear to be more than a passive consequence of the currents produced during filter feeding. However, the behavior we describe here consisted of searching for food along the bottom of the arena, with the mouthparts providing the motive force for movement through the habitat. We hereby term this behavior 'substrate browsing', because 'browsing' has been used previously for similar behaviors, and the behavior is correlated with feeding, and limited to the bottom of the container. Many larval habitats are partially filled with leaf litter and other plant detritus, and presumably the larvae would forage along this substrate rather than the bottom of the habitat under these conditions. During substrate browsing, as in gliding, larval Aedes aegypti traveled head first through the water, driven by actions of the mouthparts. However, the larvae remained along the bottom of the container, and tracks formed during substrate browsing were rarely linear, and if linear only for short distances and durations (Fig. 2). Instead, the paths typically followed broad arcs or curves, and some formed loops or circles with diameters less than body length. Velocity during substrate browsing averaged $4.5 \pm 0.38 \mathrm{~mm} \mathrm{~s}^{-1}$, but brief episodes of up to $7 \mathrm{~mm} \mathrm{~s}^{-1}$ were observed. During substrate browsing, larvae were observed briefly pausing or reversing direction for a few millimeters, while they attacked a spot on the bottom of the container with their mouthparts. Although no food was added to the arena it is likely that sensory cues were present that triggered this behavior. This suggests that the mouthpart-driven locomotion during substrate browsing is not entirely a passive consequence of 

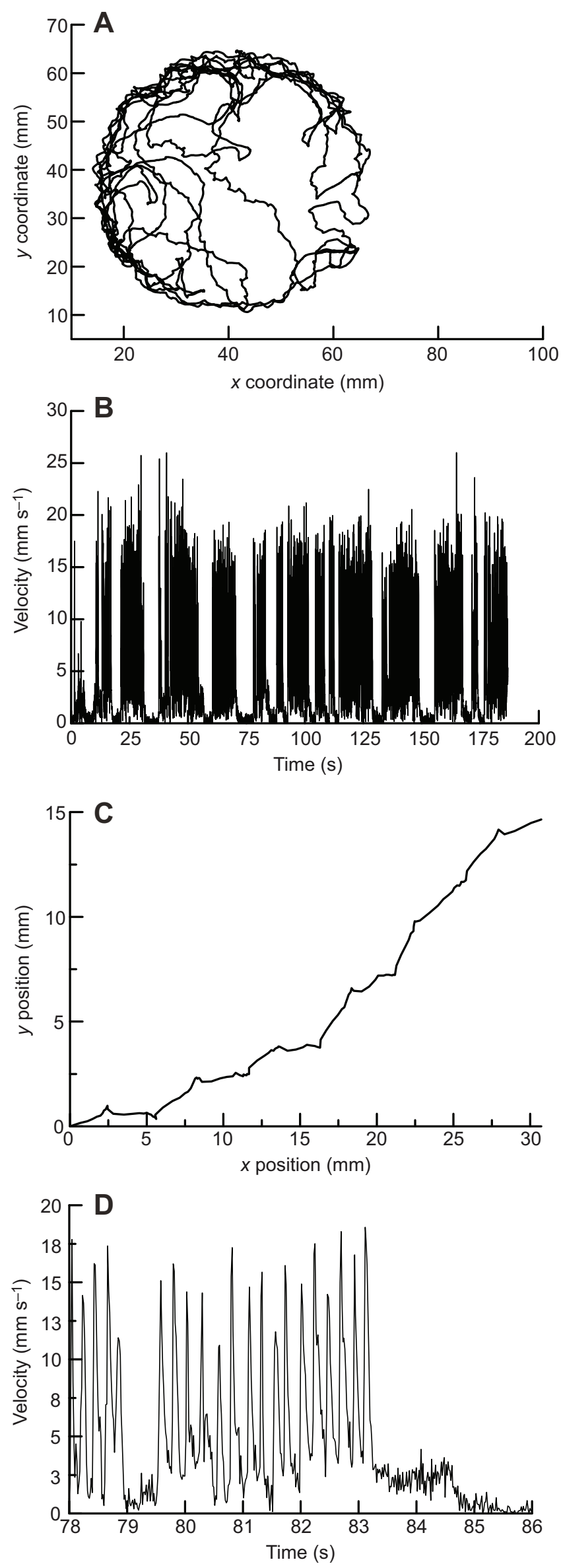

Fig. 1. Tracks of an unfed, control Aedes aegypti larva. (A) The $x-y$ coordinates of the larva during a $3 \mathrm{~min}$ trial in a $56 \mathrm{~mm}$ arena. Changes in $x-$ and $y$-coordinates over time of the approximate center of mass were obtained from a video using motion tracking software. (B) Velocity during the trial that resulted in the tracks illustrated in A. Velocity was calculated from distance traveled between successive time intervals, using the Pythagorean equation. Typical behavior consisted of bouts of wriggling, evident as a thick vertical bar created during a series of power strokes, separated by episodes of low, relatively constant velocity when the larva was stationary or performing substrate browsing. (C) The track created during a series of 12 power strokes during wriggling. The relatively linear portions of the track occur during power strokes, and the inflections and direction changes occur between strokes. (D) An $8 \mathrm{~s}$ interval from $\mathrm{C}$, expanded to show the changes in velocity during different behaviors. The graph shows a bout of wriggling, followed by a brief pause, a second bout of wriggling, a plateau from 83.2 to $84.6 \mathrm{~s}$ created during substrate browsing, and finally the velocity decreasing further as the larva becomes stationary. Note the oscillations in velocity from high values during power strokes to low velocities between power strokes during wriggling.

filter feeding but instead includes directed mouthpart-driven locomotion associated with searching for food.

During substrate browsing, curvature of the track was associated with lateral flexion of the body, with the head facing in the direction of travel (Fig. 2B). The larva showed clear directionality, and changed direction by prolonged flexion of the body so that the head pointed in the direction of travel. Any abrupt change in direction was accomplished by one or more wriggling power strokes (Fig. 2A,B).

\section{Effects of feeding status on behavior}

Feeding status significantly influenced behavior (Fig. 3, $N=14$ per treatment). Although the proportion of time spent wriggling did not change in response to feeding status, substrate browsing significantly increased in unfed larvae $(P<0.0005, t$-test $)$, whereas other behaviors including stationary filter feeding and quiescence significantly increased in fed larvae $(P<0.02, t$-test; Fig. 3$)$.

\section{Effects of paroxetine on the behavior of fed and unfed larvae} Effects of paroxetine on non-feeding behaviors

Paroxetine had no effect on the proportion of time that fooddeprived larvae spent wriggling ( $P>0.18$; Fig. 4$)$, whereas fed larvae responded to paroxetine with a doubling of the time spent wriggling $(P<0.02, N=14)$. Paroxetine did not influence the amount of time fed and food-deprived larvae performed other behaviors (food deprived: $P>0.69$, fed: $P>0.34$ ).

\section{Effects of paroxetine on feeding behavior}

Total feeding was divided into substrate browsing and SFF. Fooddeprived larvae responded to paroxetine with no change in total feeding $(P>0.19)$, whereas fed larvae responded to paroxetine with a marked decrease in total feeding $(P<0.005$; Fig. 4). Although total feeding of unfed larvae did not change in response to paroxetine, substrate browsing more than doubled $(P<0.02)$ and SFF strongly decreased $(P<0.05)$ in unfed larvae in response to paroxetine (Fig. 5). Thus, rather than altering total feeding, paroxetine caused a shift from SFF to substrate browsing in unfed larvae. In distinct contrast, fed larvae responded to paroxetine by decreasing both substrate browsing $(P<0.005)$ and SFF $(P<0.05)$ relative to controls (Fig. 5).

The effects of paroxetine on locomotion of food-derived larvae were investigated in more detail. For a subset of the data, specific episodes of wriggling or substrate browsing lasting more than a second or two could be isolated and analyzed (episodes of substrate 
Table 1. Effects of paroxetine on locomotion by food-deprived larvae

\begin{tabular}{|c|c|c|c|}
\hline & Control & Paroxetine & $P$-value $(N)$ \\
\hline Mean velocity $\left(\mathrm{mm} \mathrm{s}^{-1}\right)$ & $11.2 \pm 1.48$ & $8.1 \pm 0.85$ & $<0.05(10)$ \\
\hline Wriggling (bouts $\min ^{-1}$ ) & $7.5 \pm 1.0$ & $8.2 \pm 1.18$ & $>0.64(9)$ \\
\hline Power (strokes s ${ }^{-1}$ ) & $5.5 \pm 0.4$ & $3.3 \pm 0.32$ & $<0.0005(12)$ \\
\hline Wriggle velocity $\left(\mathrm{mm} \mathrm{s}^{-1}\right)$ & $13.1 \pm 1.74$ & $12.3 \pm 0.82$ & $>0.67(7)$ \\
\hline Browsing velocity $\left(\mathrm{mm} \mathrm{s}^{-1}\right)$ & $4.5 \pm 0.33$ & $4.3 \pm 0.26$ & $>0.63(8)$ \\
\hline
\end{tabular}

Paroxetine $\left(1 \times 10^{-5} \mathrm{~mol} \mathrm{I}^{-1}\right)$ decreases mean velocity during the entire trial, but not velocity during either wriggling or substrate browsing. As velocity is lower during substrate browsing, this suggests that the decrease in mean velocity is caused by the shift to substrate browsing. Paroxetine strongly decreases the frequency of power strokes during wriggling locomotion, yet no change is observed in the velocity during wriggling, suggesting that the mechanics of wriggling are altered by paroxetine.

Data are presented as means \pm s.e.m. Bold text indicates a significant effect of paroxetine $(P<0.05)$.

browsing were not available for several control preparations, and in other preparations wriggling locomotion occurred against the side of the arena so that appropriate episodes for analysis could not be isolated). Repeated measures were available for some trials, and for these trials an average value for that trial was used in analyses. Paroxetine strongly reduced the frequency of power strokes within bouts of wriggling $(P<0.0005, t$-test; Table 1$)$. The mean velocity over the course of the trials also significantly reduced in the
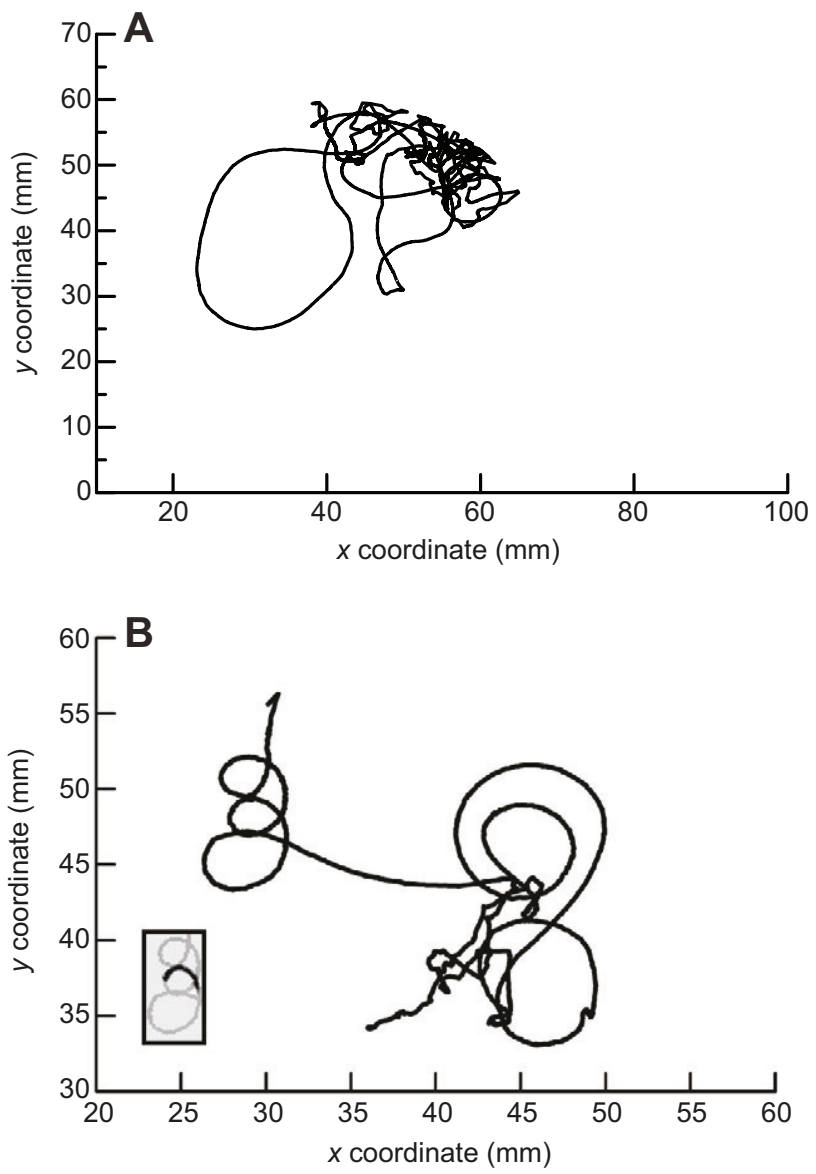

Fig. 2. Tracks of Aedes aegypti larvae showing extensive substrate browsing. (A) The tracks recorded from an unfed larva during a $3 \mathrm{~s}$ trial (B) A portion of the tracks of an unfed larva exposed to paroxetine, showing smooth tracks formed during two episodes of substrate browsing separated by erratic tracks formed during an episode of wriggling. The inset shows a copy of a portion of the track in which a low-resolution image of the larva had been superimposed onto the track using Adobe Photoshop. The head is to the left. Note the coincidence of the radius of curvature of the body and the track. presence of paroxetine (Table 1). However, when analyzed separately the velocities during individual bouts of wriggling, and during episodes of mouthpart-driven locomotion during substrate browsing, were not altered by paroxetine (Table 1).

\section{DISCUSSION}

\section{Behavior of Aedes aegypti larvae}

Under the conditions of this study the behavior of larval mosquitoes is relatively simple. Larvae typically perform bouts of wriggling of variable duration and number of power strokes, interspersed with periods during which no locomotion is observed or episodes of searching for food in which the mouthparts drive locomotion along the bottom of the container (substrate browsing). Wriggling behavior resembles that of other mosquito larvae (Strickman, 1989). Periods without locomotion include stationary SFF, in which larvae remain in one place, suspended from the surface by their respiratory siphons, but are relatively active. Flexion of the head and body associated with the direction of forward movement, and rhythmic movements of the mouthparts, are readily apparent during SFF. At other times larvae suspended from the surface film are quiescent. Quiescence is also observed for brief periods while submerged, immediately following bouts of wriggling. Mouthpart-driven movement along the bottom of the container is clearly associated

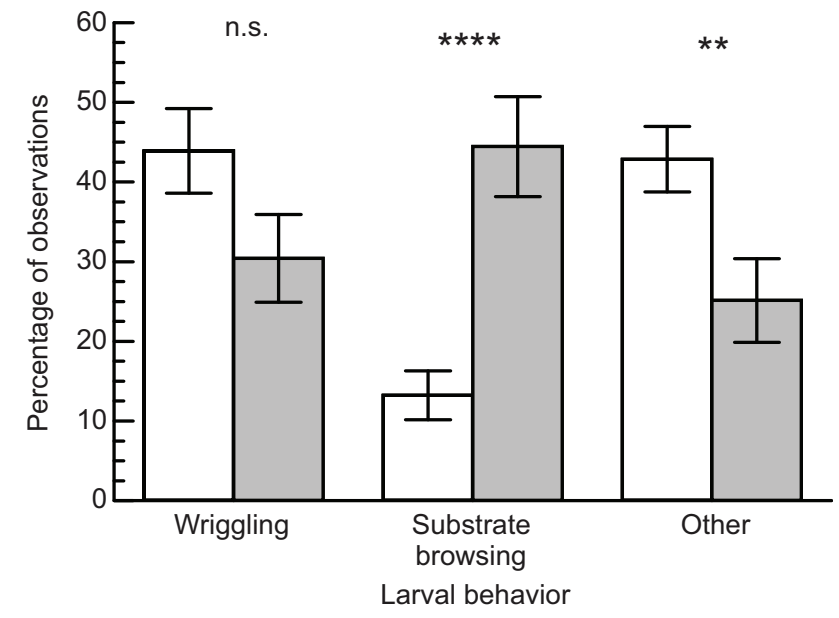

Fig. 3. Effects of feeding status on behavior in Aedes aegypti. Larvae were held with or without food for $24 \mathrm{~h}$ prior to the assay. Behavior at each $7 \mathrm{~s}$ interval during the trial was categorized from videos. Feeding status significantly influenced the proportion of observations during which larvae performed substrate browsing or performed other behaviors, but did not alter the proportion of time spent wriggling. Open bars, fed larvae; gray bars, fooddeprived larvae. Data are means \pm s.e.m. (n.s., $P>0.05 ;{ }^{* *} P<0.02$;

$\left.{ }^{* * * *} P<0.0005\right)$. 


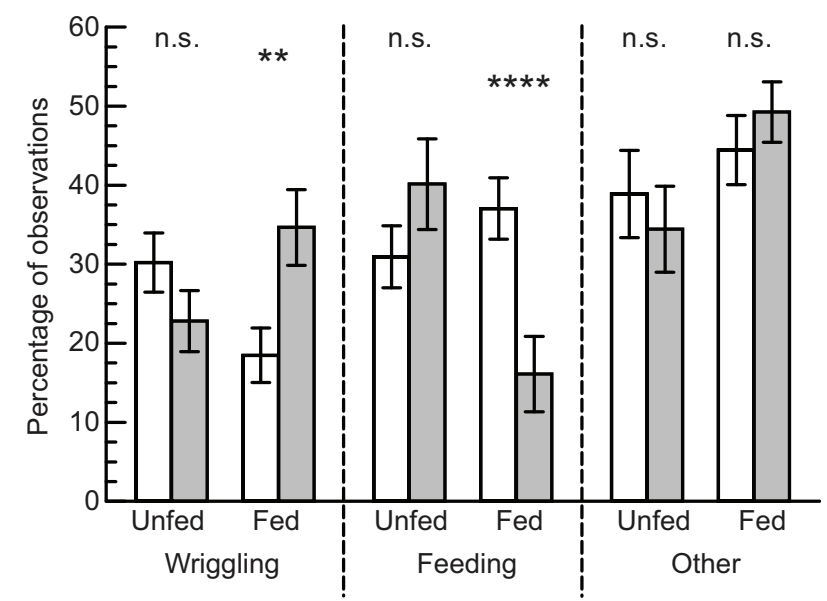

Fig. 4. Effects of paroxetine on the behavior of fed and food-deprived Aedes aegypti larvae. Larvae were held with or without food and paroxetine for $24 \mathrm{~h}$ prior to the assay. Behavior at each $7 \mathrm{~s}$ interval during the trial was categorized from videos. Wriggling: paroxetine had no effect on the incidence of wriggling by food-deprived larvae (unfed) but significantly increased wriggling by fed larvae. Feeding: paroxetine did not change the amount of time food-deprived larvae spent feeding but strongly decreased feeding rates of fed larvae. Other behaviors consisted primarily of quiescence (no locomotion or feeding). Paroxetine did not influence the proportion of time spent performing behaviors besides wriggling and feeding, by either fooddeprived or fed larvae. Open bars, control larvae; gray bars, paroxetinetreated larvae. Data are means \pm s.e.m. (n.s., $P>0.05 ;{ }^{*} P<0.05 ;{ }^{* *} P<0.02$; ${ }^{* * *} P<0.01 ;{ }^{* * *} P<0.005$ )

with feeding. As has been reported elsewhere, dense patches of labial setae act as paddles, creating currents that cause the larvae to move through the environment (Hocking, 1953; Wood and Borkent, 1989; Brackenbury, 2001). The mouthpart-driven movements that we observed along the bottom of the habitat were clearly directed by the larvae while seeking food and thus appear distinct from mouthpart-driven movements described by other authors (see below). Occasionally, the larvae were also observed briefly grooming their caudal region with their mouthparts.

\section{Substrate browsing by Aedes aegypti larvae}

Substrate browsing is hereby defined as a behavioral state in which the larvae use the mouthparts to power locomotion along the bottom of the habitat while searching for food. During this behavior, larvae move forward at relatively low velocity, with the body remaining relatively linear or maintained in a state of slight lateral flexion. The paths rarely form a straight line, but instead any path longer than a centimeter or so tends to curve, and longer tracks are meandering. Turn radii range from several times body length to significantly less than the body length (see Fig. 3C). Larval A. aegypti direct this movement by flexing the body so that the head is oriented in the direction of travel. This flexion is of a relatively low angle, is maintained for a significant period of time relative to wriggling power strokes, and does not alternate from side to side. Substrate browsing thus contains a clear directional element that is controlled by the larva, and the mouthpart-driven movements during substrate browsing are thus consistent with a distinct means of locomotion that occurs during searches of the substrate for point sources of food, rather than strictly arising as a passive consequence of currents produced during filter feeding. A number of observations support this interpretation. In several instances, larvae were observed pausing during an episode of substrate browsing and applying the mouthparts to the bottom of the container with enough force to

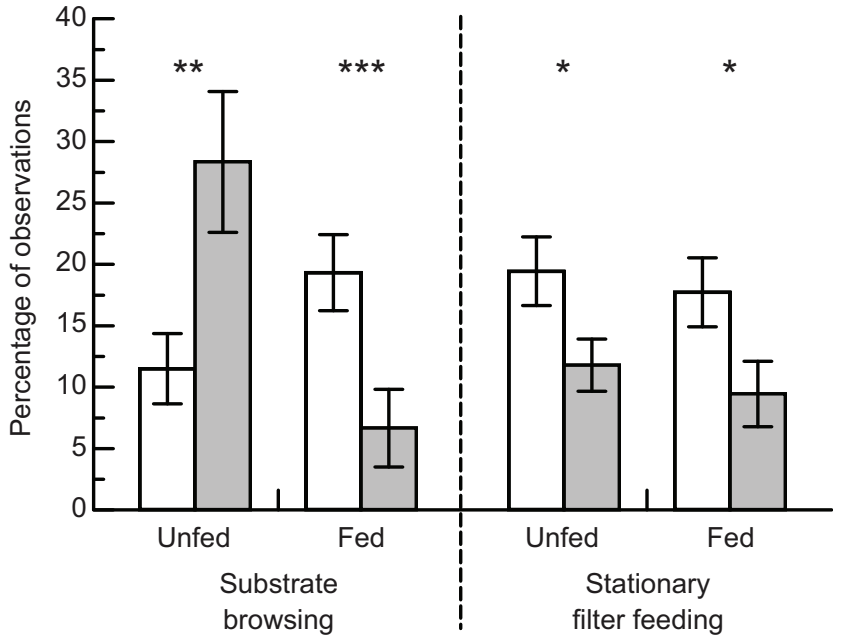

Fig. 5. Effects of paroxetine on the proportion of time spent performing distinct feeding behaviors by food-deprived and fed Aedes aegypti larvae. Behavior at each $7 \mathrm{~s}$ interval was categorized from videos. Paroxetine significantly stimulated substrate browsing and inhibited stationary surface feeding by food-deprived larvae, but inhibited both types of feeding behaviors of fed larvae. Open bars, control larvae; gray bars, paroxetine-treated larvae. Data are means \pm s.e.m. (n.s., $P>0.05$; ${ }^{*} P<0.05$; $\left.{ }^{* *} P<0.02 ;{ }^{* * *} P<0.01 ;{ }^{* * * *} P<0.005\right)$.

cause movements of the head and body. In other instances the larvae reversed direction briefly, moving backwards for short distances and re-examining a site they had just passed on the bottom of the arena. Although no food was added to the arena, these behaviors are consistent with searching for food sources that are not uniformly dispersed and are on or attached to the substrate, and are not consistent with passive movements secondary to filter feeding. We, therefore, do not consider mouthpart-driven movements, 'browsing' or 'gliding' (Hocking, 1953; Brackenbury, 2001; Christophers, 1960) to be entirely satisfactory terms for this behavior and propose 'substrate browsing', for the behavioral state in which the larvae search the bottom of the container for food, utilizing the labial brushes of the mouthparts to create motive force.

Feeding using labial brushes is limited to larval stages of the nematoceran Diptera within the superfamily Culicoidea, including mosquitoes (Culicidae), dixid midges (Dixidae) and blackflies (Simuliidae) (Wood and Borkent, 1989). Substrate foraging is likely to occur among other mosquito species, and homologous behaviors may occur in other Culicoid larvae. However, switching between the alternate feeding strategies of substrate browsing and stationary filter feeding while suspended from the surface film may be unique to larval mosquitoes. If so, the behavioral response of unfed Aedes larvae to paroxetine may also be unique to mosquito larvae. Mosquitoes also utilize other methods to obtain food, including scraping surfaces for biofilms and even consuming solid food such as dead larvae (Merritt and Cummins, 1984; Bara et al., 2014). These behaviors are predicted to occur during episodes of substrate browsing when larvae encounter nutrient sources. Intriguingly, the combination of wriggling and substrate browsing of Aedes larvae resembles the behavior of the nematode C. elegans (Shtonda and Avery, 2006; Tecott, 2007). Locomotion of the nematode is characterized by episodes of 'roaming', in which the animal moves rapidly through the habitat without feeding, and episodes of 'dwelling' in which it moves more slowly, changing direction frequently and orienting to local feeding cues. Wriggling by the mosquito is analogous to roaming, whereas substrate browsing resembles dwelling. 


\section{Effects of paroxetine on behavior}

The most striking results of the present study are the strong influence of paroxetine on behavior, and the influence of feeding status on this response. Fed larvae shift from feeding to wriggling in response to paroxetine, consistent with inhibition of appetite. In contrast, food-deprived larvae show no effects on appetite (as determined by total feeding) or wriggling, but respond to paroxetine with a shift from SFF to substrate browsing. In general, the response of larvae to food deprivation, and of food-deprived larvae to paroxetine, are very similar as both treatments stimulate substrate browsing.

Paroxetine $\left(\mathrm{Paxil}^{\mathrm{TM}}\right)$ is a serotonin-selective reuptake inhibitor (SSRI) used clinically in the treatment of depression (Dechant and Clissold, 1991). SSRIs including paroxetine alter behaviors of diverse animals, suggesting conserved pharmacological activity across taxa. Furthermore, serotonergic pathways are strongly associated with appetite, locomotion and feeding behavior in a variety of animals including leeches, gastropods, nematodes, insects and mammals (Gruninger et al., 2007; Gillette, 2006; Tecott, 2007; Halford et al., 2007; Gaudry and Kristan, 2012), and alter output from central pattern generators of the nerve cord of leeches and lamprey (Crisp and Mesce, 2003; Harris-Warrick and Cohen, 1985), actions resembling the actions of paroxetine in the larval mosquito. Thus, the effects of paroxetine on behavior of the larval mosquito are consistent with actions on serotonergic pathways involving increased synaptic serotonin through inhibition of serotonin reuptake.

The response of fed larvae to paroxetine resembles that of mammals, and most insects investigated to date, including a locust, an ant, adults of two species of flies, larval Drosophila, an aphid, and a cockroach, in all of which serotonin acts to inhibit appetite and/or feeding (Dacks et al., 2003; Kaufmann et al., 2004; Falibene et al., 2012; Neckameyer, 2010). For example, serotonin triggers a decrease in sucrose consumption by the blowfly Phormia regina, whereas the flesh fly Neobelliera bullata shows reduced feeding, and reduced electrophysiological responses to sensory input from sucrose receptors, following serotonin injection (Dacks et al., 2003; Long and Murdock, 1983). Serotonin depresses feeding, whereas low neuronal serotonin levels increase appetite of Drosophila larvae (Neckameyer, 2010). In contrast, adults of the mosquito Aedes triseriatus respond to depletion of serotonin levels with a decrease in the amount of blood taken during feeding and in numbers of feeding episodes, but with no change in host-seeking behavior, suggesting that serotonin stimulates feeding (Novak and Rowley, 1994). The apparent difference in the roles of serotonin on appetite of larval and adult mosquitoes merits further study. The role of serotonin in larval mosquitoes also resembles that in the nematode C. elegans, in which the SSRI fluoxetine stimulates, and serotonin receptor antagonists suppress, feeding-related dwelling behavior (Sawin et al., 2000; Tecott, 2007), a behavior that appears analogous to substrate browsing of $A$. aegypti larvae. It is not clear whether these similarities result from highly conserved regulation of behavioral processes by serotonin within Ecdysozoa, or are convergent in these two groups. The systems are not exactly the same, because unlike C. elegans, in which the response to fluoxetine is only apparent in the presence of bacteria (Sawin et al., 2000), changes in behavior of larval A. aegypti in response to paroxetine were observed in the absence of food. In contrast, serotonin stimulates appetite in leeches and molluscs (members of the Lophotrochozoa) (Tecott, 2007; Gaudry and Kristan, 2012) suggesting a major evolutionary change since the common ancestor of these two major lineages of protostomes. In leeches and molluscs,
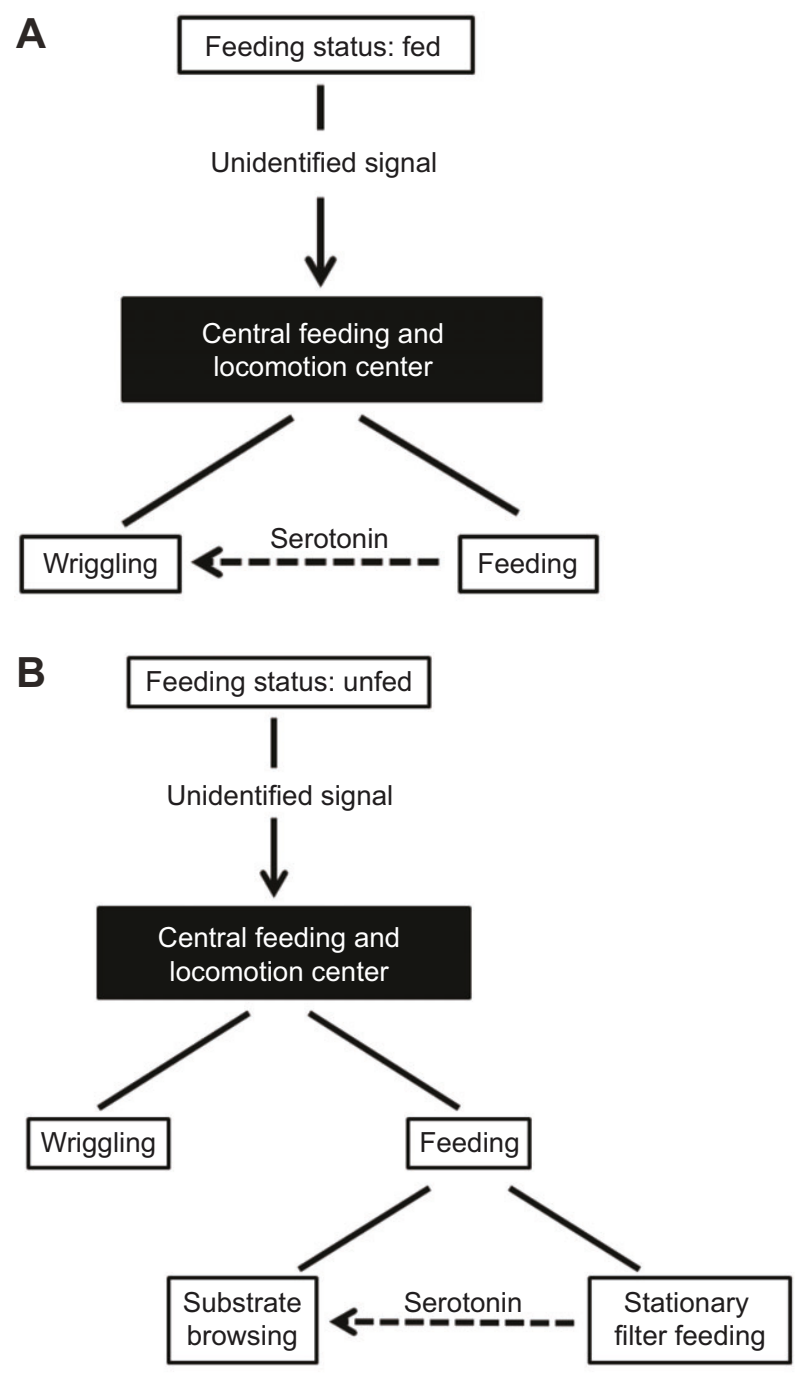

Fig. 6. Hypothetical regulation of the feeding center by serotonin. The switch between feeding and wriggling behaviors originates in a central feeding and locomotion center in the brain. This center is set into discrete functional states by unidentified sensory signals reflecting feeding (or nutritional) status. Serotonergic pathways acting on this center result in different behavioral outputs depending on the feeding status. The unidentified signal reflecting feeding status is elevated in either the fed or unfed state. (A) The fed state. Serotonin shifts behavioral output from feeding to wriggling. Independent regulation of two distinct feeding modes is not apparent as both feeding modes are reduced in response to serotonergic input. (B) The unfed state. Serotonin shifts behavior from stationary filter feeding at the water surface to substrate browsing, rather than from feeding to wriggling as occurs in the fed state. Independent regulation of two distinct feeding modes, substrate browsing and stationary filter feeding, is revealed.

satiation is primarily driven by sensory input from stretch receptors whereas in mammals peptide hormones and neurotransmitters mediate satiety in response to endocrine signals from the gut and from nutrient stores (Gillette, 2006; Halford et al., 2007; Gaudry and Kristan, 2012). The signals driving satiation, and triggering the change in the feeding centers from the fed to the unfed state, in the larval mosquito are unknown. Neuropeptide $Y$ and insulin-like peptide are candidates (Nässel and Wegener, 2011).

The central feeding centers switch between satiated and nonsatiated states that differ in their responses to paroxetine (Fig. 6A,B). Shifts among distinct feeding modes in response to serotonergic pathways, similar to the shifts between stationary filter 
feeding and substrate browsing observed in larvae Aedes, have not been described in other species. Is this response unique to mosquito larvae? The distinct behavioral responses of fed and food-deprived larvae show that these feeding modes are regulated independently, suggesting that they may provide distinct nutritional resources. Modulation of feeding centers by serotonin in vivo might cause food-deprived larvae to actively seek out nutrient sources on the substrate when the amount or quality of suspended material in the water column is insufficient to provide adequate nourishment. If so, paroxetine may mimic responses to more extreme starvation than caused by the experimental protocol used here. The role of serotonin on appetite of larval and adult mosquitoes merits further study.

\section{Effects of paroxetine on wriggling locomotion}

In the leech, swimming is controlled in a top-down manner, with the decision to swim residing in the head brain, whereas the output to the muscles carrying out the muscular contractions originates within the nerve cord (Esch et al., 2002; Crisp and Mesce, 2003). In the leech, application of serotonin to the head brain inhibits swimming, whereas application to the nerve cord stimulates swimming (Crisp and Mesce, 2003). Serotonin also acts on central pattern generators of the dorsal nerve cord of the lamprey (Harris-Warrick and Cohen, 1985). Similarly, we show here that serotonin influences the decision of the larval mosquito to wriggle or feed, consistent with actions in the brain, and also acts on the stroke frequency within bouts of wriggling, consistent with actions on downstream central pattern generators. The presence of serotonin-modulated efferent signals to swimming muscles of lamprey and larval mosquito (Crisp and Mesce, 2003; Harris-Warrick and Cohen, 1985; present study) suggest highly conserved neural pools. However, the wriggling of mosquito larvae is a derived character shared by the larval stages of a few dipteran taxa. Thus, the actions of the central pattern generators that drive locomotion in the mosquito larva and lamprey appear convergent. We also did not observe any effect of paroxetine on velocity during wriggling, despite a significant change in power stroke frequency. This suggests that the biomechanics of power strokes are influenced by paroxetine.

In addition to its role in regulating feeding-related behaviors and appetite, serotonin is clearly a hormone involved in regulation of the physiological responses to feeding of insects, including the true bug Rhodnius prolixus (Barrett and Orchard, 1990; Lange et al., 1989; Maddrell et al., 1991; Maddrell et al., 1993; Orchard, 2006). Serotonin is also involved in regulation of peripheral tissues in larval $A$. aegypti, but the role of serotonin in the physiological responses of larval A. aegypti to feeding are less clear than in Rhodnius. Serotonin stimulates acid/base transport of the larval mosquito midgut, and fluid secretion by the Malpighian tubules (Clark and Bradley, 1996; Clark and Bradley, 1997; Clark et al., 1999; Onken et al., 2008; Onken and Moffett, 2009); actions likely to be associated with feeding. However, Clark and Bradley detected no changes in hemolymph serotonin levels of larval A. aegypti in response to feeding status (Clark and Bradley, 1997). It is possible that a different feeding or assay protocol than used by Clark and Bradley would trigger detectable serotonin release, or that serotonin regulates midgut transport by synaptic or local control rather than by neuroendocrine mechanisms and thus does not increase in the hemolymph in response to feeding activity.

\section{Summary}

The results of the present study support an important role of serotonergic pathways in feeding behavior of larval A. aegypti. The distinct responses of fed and unfed larvae to the same drug treatment suggest that the feeding centers are modulated by input from signals related to feeding or nutritional status, altering their responses to stimulation by serotonergic pathways. Such signals are currently unknown in the mosquito and should be explored. The similarities between $C$. elegans, in which fluoxetine shifts behavior from roaming to dwelling (Sawin et al., 2000; Tecott, 2007), and unfed Aedes larvae, in which paroxetine increases substrate browsing, a behavior similar to dwelling, suggest that the link between serotonergic pathways and foraging behavior may be highly conserved, at least among Ecdysozoa. Furthermore, investigators working with other model systems addressing appetite regulation by serotonergic pathways should consider the possibility that feeding status may influence the responses of their systems to manipulation of serotonergic pathways. Finally, serotonergic drugs prescribed as antidepressants are appearing in waste-water and waterways at physiologically relevant concentrations (Brooks et al., 2005; Conners et al., 2009; Kwon and Armbrust, 2006; Metcalfe et al., 2010). The results of the current study suggest that environmental pollution with serotonergic drugs may influence the appetite, and thus growth and development, of freshwater insects. These insects are crucial for converting plant biomass to animal biomass in aquatic habitats, and disruption of their appetite and feeding behavior may have repercussions throughout food webs.

\section{MATERIALS AND METHODS \\ Mosquitoes}

Aedes aegypti (L.) larvae used in this study were derived from eggs obtained from wild-caught mosquitoes collected in Palm Beach County, Florida, in 2008. Larvae were reared in deionized water on pulverized Tetramin tropical fish flakes (Tetra Holding, Inc., Blacksburg, VA, USA). Fourth instar larvae were used in experiments.

\section{Experimental design of behavioral assays}

During initial observations, the relative amount of time spent performing different behaviors was found to vary from one experiment to the next (compare Figs 3 and 5). The causes of these differences were not examined, but all comparisons between treatment groups were therefore performed using data collected during the same session, with larvae from the same cohort, and the different treatment groups within an experiment were assayed one replicate at a time in series until all replicates of each treatment group were obtained. Two separate experiments were performed, one to determine the effects of feeding status on behavior (Fig. 3), and the second to determine separately the effects of paroxetine on behavior of fed and unfed larvae (Figs 4, 5).

Paroxetine hydrochloride hemihydrate (Sigma-Aldrich, St Louis, MO, USA) was dissolved in water and added to wells at a final concentration of $2 \times 10^{-5} \mathrm{~mol}^{-1}$, a concentration previously determined to cause no fatalities under similar conditions. Larvae were placed in groups of four or five in $5 \mathrm{ml}$ of deionized water in the wells of six-well plates. One group of larvae was maintained without food for the subsequent $24 \mathrm{~h}$. Half of this group was exposed to paroxetine for $24 \mathrm{~h}$, while the other half received no paroxetine. A second group of larvae was isolated at the same time, but provided with food upon isolation, and fed again the next morning (approximately $3 \mathrm{~h}$ prior to initiating assays). Half of this group received paroxetine upon isolation and the remainder received no paroxetine. Prior to videotaping, larvae were rinsed in deionized water. Larvae were transferred individually to a $57.25 \mathrm{~mm}$ inner diameter Petri dish containing $15 \mathrm{ml}$ deionized water, and positioned on a light box with dim light from indirect LED lighting diffused through paper, from below the arena. They were allowed to adjust to their new environment for $1 \mathrm{~min}$, and then videotaped. No larvae died during exposure to paroxetine or in control treatments.

\section{Video recordings}

Behavior was recorded at room temperature $\left(22^{\circ} \mathrm{C}\right)$ using a Canon EOS 7D digital camera with a $100 \mathrm{~mm} \times 2.8$ IS lens (Canon USA, Inc., Lake 
Success, NY, USA). Movements of each larva were recorded for at least $3 \mathrm{~min}$ at a resolution of $640 \times 480$ pixels and a frame rate of 60 frames s $\mathrm{s}^{-1}$. Movies were converted to avi files using Prism (Prism Software Corp., http://www.prismsoftware.com), then imported into Swistrack motion tracking software (Swistrack is available as freeware at http://sourceforge.net/projects/swistrack/).

\section{Swistrack output}

Output from Swistrack included a column of video frame numbers (frameline) and the corresponding $x$ - and $y$-coordinates of the larva in each frame. A timeline was generated from the frameline using the frame numbers and the 60 frames s$^{-1}$ recording speed. Static points a known distance apart in the $x$ - and $y$-axes, videotaped at the same focal length as used in experiments, were used to calibrate the distance between the positional coordinates in the Swistrack output. The distance traveled by larvae between consecutive recorded $x-y$ coordinates was determined from the distance traveled in the $x$ - and $y$-axes, using the Pythagorean equation to determine the length of the hypotenuse of the triangle.

\section{Determination of the proportion of time spent performing different} behaviors

Using videos, the behavior of each larva was documented at $7 \mathrm{~s}$ intervals.

\section{Velocity of locomotion}

Distance between consecutive $x-y$ coordinates was divided by the time increment to give an estimate of instantaneous velocity, and the total distance traveled during the trial was summed and divided by duration of the trial to establish mean velocity. To determine the velocity during wriggling locomotion, we identified periods of wriggling locomotion during which the larva crossed the central area of the arena and the direction of travel in the $x$ - and $y$-coordinates remained constant for at least $1 \mathrm{~s}$. The sum of the individual distances calculated from one set of positional coordinates to the next during the episode gave total distance traveled, and the difference in recorded time between the beginning and end of the episode was used to calculate the velocity. For velocity during substrate browsing, periods of substrate browsing of at least $2 \mathrm{~s}$ duration were identified, and velocity calculated as for wriggling locomotion. As episodes meeting these parameters were not present in all videos the $N$-values of these experiments are less than those in the other analyses.

\section{Statistics}

Comparison between controls and treatment groups, or between fed and unfed larvae, of the proportion of time spent performing a specific behavior were made using Student's $t$-test (PsiPlot, Poly Software Intl, Pearl River, NY, USA)

\section{Acknowledgements}

The authors thank Peter Bushnell for assistance with setting up Swistrack analyses, Michael Reiskind for mosquito eggs, Jeffrey Bara for a critical reading of the manuscript, and anonymous reviewers for helpful suggestions.

\section{Competing interests}

The authors declare no competing financial interests.

\section{Author contributions}

All authors participated in experimental design, data collection and data analysis. T.M.C. was primarily responsible for writing the article.

\section{Funding}

This work was solely supported by the Indiana University South Bend.

\section{References}

Anstey, M. L., Rogers, S. M., Ott, S. R., Burrows, M. and Simpson, S. J. (2009). Serotonin mediates behavioral gregarization underlying swarm formation in desert locusts. Science 323, 627-630.

Bara, J. J., Clark, T. M. and Remold, S. K. (2014). Utilization of larval and pupal detritus by Aedes aegypti and Aedes albopictus. J. Vector Ecol. (in press).

Barrett, M. and Orchard, I. (1990). Serotonin-induced elevation of CAMP levels in the epidermis of the blood-sucking bug, Rhodnius prolixus. J. Insect Physiol. 36, 625633.

Brackenbury, J. (2001). Locomotion through use of the mouth brushes in the larva of Culex pipiens (Diptera: Culicidae). Proc. Biol. Sci. 268, 101-106.
Brooks, B. W., Chambliss, C. K., Stanley, J. K., Ramirez, A., Banks, K. E., Johnson, R. D. and Lewis, R. J. (2005). Determination of select antidepressants in fish from an effluent-dominated stream. Environ. Toxicol. Chem. 24, 464-469.

Christophers, S. R. (1960). Aedes Aegypti (L.), The Yellow Fever Mosquito; Its Life History, Bionomics and Structure. Cambridge: Cambridge University Press.

Clark, T. M. and Bradley, T. J. (1996). Stimulation of Malpighian tubules from larval Aedes aegypti by secretagogues. J. Insect Physiol. 42, 593-602.

Clark, T. M. and Bradley, T. J. (1997). Malpighian tubules of larval Aedes aegypti are hormonally stimulated by 5-hydroxytryptamine in response to increased salinity. Arch. Insect Biochem. Physiol. 34, 123-141.

Clark, T. M., Koch, A. and Moffett, D. F. (1999). The anterior and posterior 'stomach' regions of larval Aedes aegypti midgut: regional specialization of ion transport and stimulation by 5-hydroxytryptamine. J. Exp. Biol. 202, 247-252.

Conners, D. E., Rogers, E. D., Armbrust, K. L., Kwon, J.-W. and Black, M. C. (2009). Growth and development of tadpoles (Xenopus laevis) exposed to selective serotonin reuptake inhibitors, fluoxetine and sertraline, throughout metamorphosis. Environ. Toxicol. Chem. 28, 2671-2676.

Crisp, K. M. and Mesce, K. A. (2003). To swim or not to swim: regional effects of serotonin, octopamine and amine mixtures in the medicinal leech. J. Comp. Physiol. A 189, 461-470.

Dacks, A. M., Nickel, T. and Mitchell, B. K. (2003). An examination of serotonin and feeding in the flesh fly Neobellieria bullata (Sarcophagidae: Diptera). J. Insect Behav. $16,1-21$.

Dechant, K. L. and Clissold, S. P. (1991). Paroxetine. A review of its pharmacodynamic and pharmacokinetic properties, and therapeutic potential in depressive illness. Drugs 41, 225-253.

Esch, T., Mesce, K. A. and Kristan, W. B., Jr (2002). Evidence for sequential decision making in the medicinal leech. J. Neurosci. 22, 11045-11054.

Falibene, A., Rössler, W. and Josens, R. (2012). Serotonin depresses feeding behaviour in ants. J. Insect Physiol. 58, 7-17.

Gaudry, Q. and Kristan, W. B., Jr (2012). Decision points: the factors influencing the decision to feed in the medicinal leech. Front. Neurosci. 6, 101.

Gillette, R. (2006). Evolution and function in serotonergic systems. Integr. Comp. Biol. 46, 838-846.

Gruninger, T. R., LeBoeuf, B., Liu, Y. and Garcia, L. R. (2007). Molecular signaling involved in regulating feeding and other motivated behaviors. Mol. Neurobiol. 35, 1 19.

Guler, Y. and Ford, A. T. (2010). Anti-depressants make amphipods see the light. Aquat. Toxicol. 99, 397-404.

Halford, J. C. G., Harrold, J. A., Boyland, E. J., Lawton, C. L. and Blundell, J. E. (2007). Serotonergic drugs: effects on appetite expression and use for the treatment of obesity. Drugs 67, 27-55.

Harris-Warrick, R. M. and Cohen, A. H. (1985). Serotonin modulates the central pattern generator for locomotion in the isolated lamprey spinal cord. J. Exp. Biol. $116,27-46$.

Hocking, B. (1953). Notes on the activities of Aedes larvae. Mosq. News 13, 77-81

Huber, R., Smith, K., Delago, A., Isaksson, K. and Kravitz, E. A. (1997). Serotonin and aggressive motivation in crustaceans: altering the decision to retreat. Proc. Natl. Acad. Sci. USA 94, 5939-5942.

II-Han, J., Janes, T. and Lukowiak, K. (2010). The role of serotonin in the enhancement of long-term memory resulting from predator detection in Lymnaea. $J$. Exp. Biol. 213, 3603-3614.

Jackson, N. (1953). Observations on the feeding habits of a predaceous mosquito larva, Culex (Lutzia) tigripes Grandpré and Charmoy. Proceedings of the Royal Entomological Society of London 28, 153-159.

Kaufmann, L., Schürmann, F., Yiallouros, M., Harrewijn, P. and Kayser, H. (2004). The serotonergic system is involved in feeding inhibition by pymetrozine. Comparative studies on a locust (Locusta migratoria) and an aphid (Myzus persicae). Comp. Biochem. Physiol. 138C, 469-483.

Kwon, J.-W. and Armbrust, K. L. (2006). Laboratory persistence and fate of fluoxetine in aquatic environments. Environ. Toxicol. Chem. 25, 2561-2568.

Lange, A. B., Orchard, I. and Barrett, F. M. (1989). Changes in hemolymph serotonin levels associated with feeding in the blood-sucking bug, Rhodnius prolixus. J. Insect Physiol. 35, 393-397, 399.

Long, T. F. and Murdock, L. L. (1983). Stimulation of blowfly feeding behavior by octopaminergic drugs. Proc. Natl. Acad. Sci. USA 80, 4159-4163.

Luedtke, S., O'Connor, V., Holden-Dye, L. and Walker, R. J. (2010). The regulation of feeding and metabolism in response to food deprivation in Caenorhabditis elegans. Invert. Neurosci. 10, 63-76.

Maddrell, S. H. P., Herman, W. S., Mooney, R. L. and Overton, J. A. (1991). 5Hydroxytryptamine: a second diuretic hormone in Rhodnius prolixus. J. Exp. Biol. $156,557-566$.

Maddrell, S. H. P., Herman, W. S., Farndale, R. W. and Riegel, J. A. (1993). Synergism of hormones controlling epithelial fluid transport in an insect. J. Exp. Biol. 174, 65-80.

Merritt, R. W. and Cummins, K. W. (1984). An Introduction to the Aquatic Insects, Second edition. Dubuque, IA: Kendall/Hunt Publishing Company.

Metcalfe, C. D., Chu, S., Judt, C., Li, H., Oakes, K. D., Servos, M. R. and Andrews, D. M. (2010). Antidepressants and their metabolites in municipal wastewater, and downstream exposure in an urban watershed. Environ. Toxicol. Chem. 29, 79-89.

Nässel, D. R. and Wegener, C. (2011). A comparative review of short and long neuropeptide F signaling in invertebrates: Any similarities to vertebrate neuropeptide Y signaling? Peptides 32, 1335-1355.

Neckameyer, W. S. (2010). A trophic role for serotonin in the development of a simple feeding circuit. Dev. Neurosci. 32, 217-237. 
Novak, M. G. and Rowley, W. A. (1994). Serotonin depletion affects blood-feeding but not host-seeking ability in Aedes triseriatus (Diptera: Culicidae). J. Med. Entomol. 31, 600-606.

Onken, H. and Moffett, D. F. (2009). Revisiting the cellular mechanisms of strong luminal alkalinization in the anterior midgut of larval mosquitoes. J. Exp. Biol. 212, 373-377.

Onken, H., Moffett, S. B. and Moffett, D. F. (2008). Alkalinization in the isolated and perfused anterior midgut of the larval mosquito, Aedes aegypti. J. Insect Sci. 8, 1 20.

Orchard, I. (2006). Serotonin: a coordinator of feeding-related physiological events in the blood-gorging bug, Rhodnius prolixus. Comp. Biochem. Physiol. 144A, 316-324.

Sawin, E. R., Ranganathan, R. and Horvitz, H. R. (2000). C. elegans locomotory rate is modulated by the environment through a dopaminergic pathway and by experience through a serotonergic pathway. Neuron 26, 619-631.

Shtonda, B. B. and Avery, L. (2006). Dietary choice behavior in Caenorhabditis elegans. J. Exp. Biol. 209, 89-102.
Strickman, D. (1989). Biosystematics of larval movement of Central American mosquitoes and its use for field identification. J. Am. Mosq. Control Assoc. 5, 208218.

Tecott, L. H. (2007). Serotonin and the orchestration of energy balance. Cell Metab. 6 , 352-361.

Thamm, M., Balfanz, S., Scheiner, R., Baumann, A. and Blenau, W. (2010). Characterization of the 5-HT1A receptor of the honeybee (Apis mellifera) and involvement of serotonin in phototactic behavior. Cell. Mol. Life Sci. 67, 2467-2479.

Waggoner, L. E., Zhou, G. T., Schafer, R. W. and Schafer, W. R. (1998). Control of alternative behavioral states by serotonin in Caenorhabditis elegans. Neuron 21, 203-214.

Wood, D. M. and Borkent, A. (1989). Phylogeny and classification of the Nematocera In Manual of Nearctic Diptera, Vol. 3 (ed. J. F. McAlpine), Monograph 32. Ottawa, ON: Biosystematics Research Centre.

Yee, D. A., Kesavaraju, B. and Juliano, S. A. (2004). Larval feeding behavior of three co-occurring species of container mosquitoes. J. Vector Ecol. 29, 315-322. 